\title{
Analysis of the extreme temperature's effect on the low-velocity impact behaviour and damage evolution in sandwich components
}

\author{
Ilaria Papa, Luca Boccarusso, Davide Mocerino and Antonio Langella
}

\begin{abstract}
Ilaria Papa. Department of Chemical, Materials and Production Engineering, University of Naples Federico II, P.le Vincenzo Tecchio 80, 80125 Naples, Italy

Corresponding author: ilaria.papa@unina.it

Luca Boccarusso. Department of Chemical, Materials and Production Engineering, University of Naples Federico II, P.le Vincenzo Tecchio 80, 80125 Naples, Italy

Davide Mocerino. Department of Chemical, Materials and Production Engineering, University of Naples Federico II, P.le Vincenzo Tecchio 80, 80125 Naples, Italy

Antonio Langella. Department of Chemical, Materials and Production Engineering, University of Naples Federico II, P.le Vincenzo Tecchio 80, 80125 Naples, Italy

Abstract. Literature reports several works concerning the impact response of composites reinforced with woven fibers and the mechanisms involved in impact energy dissipation. However, even if composites' structures are subjected to various environmental conditions during their service life, few papers are concerned about the impact behaviour at shallow temperatures. Very few discuss sandwich components. In this paper, sandwich composites' low-velocity impact behaviour constructed from carbon fibre reinforced face sheets surrounding a polyvinyl chloride core has been tested. Impact tests at penetration and indentation $(U=5,7.5,12,10$ and 15J) at room temperature and $-55^{\circ} \mathrm{C},+70{ }^{\circ} \mathrm{C}$ were carried out. After each impact test, indentation depth and ultrasound analysis measurements have been implemented to learn the damage modes of the tested samples. The results provided a useful indication on the extreme temperature's influence on both impact properties and damage evolution.
\end{abstract}

Keywords. Low Velocity Impact, Sandwiches, Extreme Temperatures, Damage, Ultrasound

\section{Introduction}

Since composite materials offer numerous advantages over conventional materials, they have been widely used in various engineering applications such as in transportation, aerospace and marine. Even if the static performance of sandwich composites has been profoundly analysed, the dynamic is important too. The response of the structures to impact events is crucial in the project of solid constructions. The impact behaviour of composites has limited their use in specific applications. Tools falling on a composite outward, rocks impact a vehicle and ballistic events on aeroplane plates produce impact scenarios. Several studies have been developed to comprehend composites reaction to such loading in terms of damage and residual strength. The ability of a structure to job after a particular impact damage entity is of crucial importance. However, due to their lack of through-the-thickness reinforcement, one of the primary raw backs of composite materials is their relatively poor impact loading resistance. The impact loading may induce matrix cracking, fibre breakage and subsurface delamination. But the in-service conditions are not always likely to occur at each temperature and, especially, at extreme ones. However, the supply of carbon fibre composites (CFRP) often cannot catch up with demand; therefore, they exhibit a high cost. Sandwich structural composites are usually suggested to reduce cost and construct lightweight structures with high mechanical load capacity. Composite sandwich panels composed of a thick low density core can improve different structural properties such as stiffness, strength, sound insulation, low heat transfer, and high impact energy absorption [1]. Today, this type of structures is used in aerospace, marine, transport, and civil industries [2]. Low resistance under low-velocity impact loading is one of the important vulnerabilities of composite sandwich panels. Hence, to improve their performance, it is necessary to comprehensively investigate composite sandwich panels' behaviour under these loads conditions and investigate the 
Analysis of the extreme temperature's effect on the low-velocity impact behaviour and d...

damage that in composite laminate is often barely visible. Several papers have been developed recently to illustrate the damage acceptance and residual strength of different sandwich composites structures after low- and high-velocity impacts. Reddy et al. [3] considered quasi-static and dynamic effects and suggested a dynamic improvement feature to test the difference between static and dynamic loading. Vaidya et al. [4] have conducted several studies to analyse the impact damage tolerance of sandwich composites structures characterized by advanced core constructions. Many authors studied the changes in the material properties as a function of temperature [5]. Anyway, even if composites' structures are subjected to various environmental conditions during their service life, few papers are concerned about the impact behaviour at extremely low and high temperatures, and very few discuss sandwich components [6].

In this study, sandwich composites' low-velocity impact behaviour up to penetration and at different increasing energy levels at room temperature, $\mathrm{T}_{\mathrm{a}}$, and $-55^{\circ} \mathrm{C},+70^{\circ} \mathrm{C}$ were carried out. Indentation depth and ultrasound (US) analysis measurements have been performed to obtain information on the impacted samples' damage mechanisms. The results provided a useful indication on the extreme temperature's effect on both impact properties and damage start and propagation. The extreme temperature exposure creates a larger damage zone. The damage results in a greater damage in the face sheets or the core material dependent on the temperature tested.

\section{Materials and methods}

Sandwich composites samples tested are composed of polyvinyl chloride (PVC) core enclosed in an upper skin composed of fabric at 0/90 ( TC-EP250TU2-1/CF200T2/2-45\%) and unidirectional at $0^{\circ}$, and a lower skin composed of fabric at 0/90 ( TC-EP250TU2-1/CF200T2/2-45\%) and a biaxial at \pm 45 (TC-EP250TU/2-1/CFBX+/-45-200-47\%) symmetric, $[0 / 90, \pm 45] \mathrm{s}$. The panels were realized with a vacuum impregnation method using carbon pre impregnated laminates; first of all carbon laminates were stacked in desired sequences, after a PVC foam was placed inside and other carbon laminates were put over in order to have the desired lamination sequences. All was posed in a bag with a vacuum pump and using a temperature of $100^{\circ}$ for the foam curing to obtain a tested specimen with a dimension of $200 \mathrm{~mm} \times 200 \mathrm{~mm}$ for the entire panel and a dimension of $80 \mathrm{mmx} 80 \mathrm{~mm}$ for the PVC foam. The thickness of the results of the specimen of $4.2 \mathrm{~mm}$ in the centre.

\subsection{Characterization techniques}

Impact tests were conducted using a falling dart testing machine Ceast Instron, equipped with the thermostatic chamber and anti-rebound system. The impactor has a hemispherical shape with a diameter equal to $19.8 \mathrm{~mm}$. The specimen impacted has been positioned in the testing area on a dedicated fixture with dimensions according to ASTM D7136M international. The composites' laminates have been tested at penetration and three energy levels - 7.5, 10 and $12.5 \mathrm{~J}$ respectively - by keeping the impact mass constant and varying the drop height position. Moreover, low-velocity impact tests were performed at room temperature, Ta, (approx. $25^{\circ} \mathrm{C}$ ) and at $-55^{\circ}$ and $70^{\circ} \mathrm{C}$. For testing at temperatures lower than room one, the samples were soaked at that temperature for a minimum of 45 minutes. The achievement of uniform temperature throughout samples length was assessed using a high-resolution infrared camera, Avio RX500E Pro and confirmed by a thermocouple applied on the specimen. After, testing was performed in the climatic chamber at $\mathrm{T}=-55^{\circ} \mathrm{C}$ and $\mathrm{T}=70^{\circ} \mathrm{C}$ as for standard. The updating of the MTS and Instron machines by a thermal chamber allowed tests at high temperatures and low-temperature thanks to liquid nitrogen. Fifteen minutes were necessary to get -50 ${ }^{\circ} \mathrm{C}$. The cooling system involves the supply of liquid nitrogen from the commercially available liquid nitrogen tank through a control valve, which releases the evaporated liquid nitrogen into the environment chamber. At each of the impact energy and temperature combinations, a set of three specimens was tested. After each impact tests, the damage in terms of indentation depth was evaluated using 3D optical profiler combining confocal by Sensofar S-Neox coupled with a lens 5X zoom and an acquisition area of $18 \mathrm{~mm} \mathrm{X} 18 \mathrm{~mm}$. After impact tests, the delaminated area was evaluated by ultrasound (US) technique using a Multi 2000 Pocket system and a probe array, $\mathrm{f}=5 \mathrm{MHz}$. The C-scan acquisition 
is obtained using the US apparatus in the form of reflection. Remembering that the C-scan is the reconstruction of the material status ply by ply reported in one plane, it is possible to know the damage's shape and extension. Thanks to a Cad software the damaged area can be evaluated. During the acquisition, the pulse Echo technique was adopted, and a propagation velocity equal to $3000 \mathrm{~mm} / \mathrm{s}$ was registered.

\section{Results and discussions}

Figure 1 shows the load displacement curves at penetration for all temperatures tested. As clearly indicate in table 1 , for the room temperature the highest maximum load, F, and impact energy, $U_{p}$, are detected.

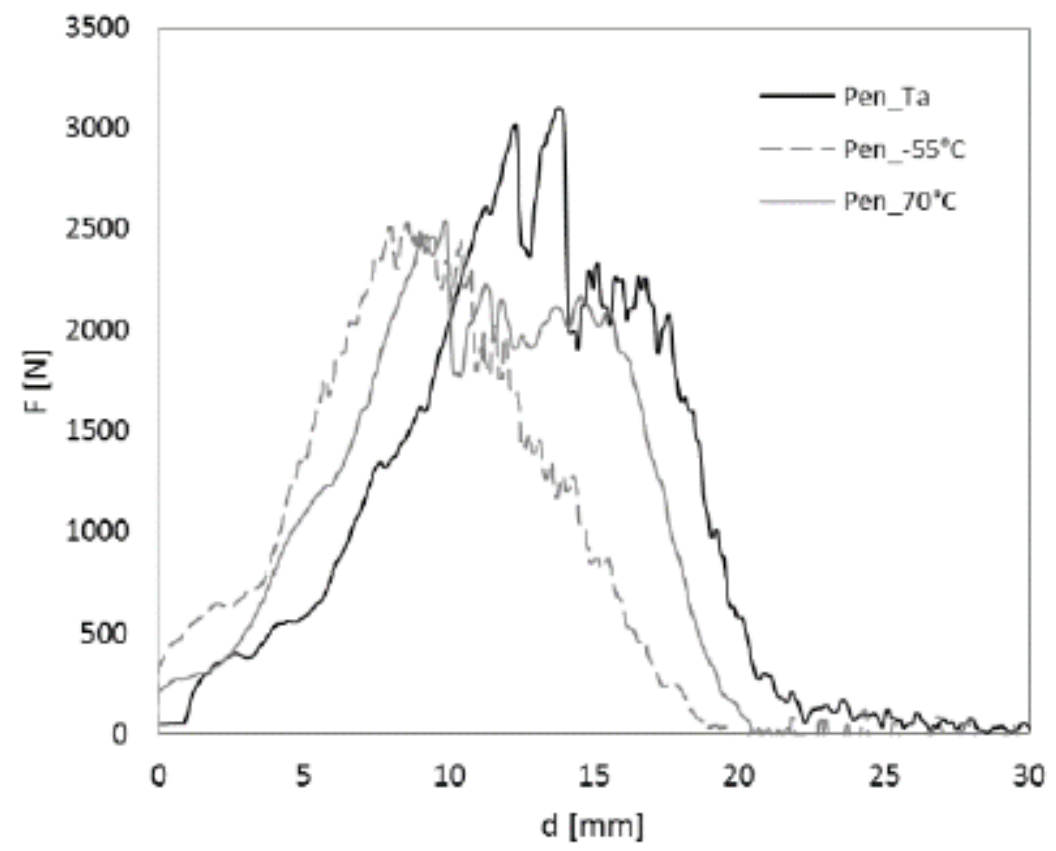

Fig. 1. Load-displacement curves at penetration for the three tested temperatures

A similar maximum load is shown at $-55^{\circ} \mathrm{C}$ and $70^{\circ} \mathrm{C}$ temperature even if, for the latter, higher impact energy is needed to penetrate the sample due to the rigidity increasing at a lower temperature.

Table 1. Impact parameters at penetration

\begin{tabular}{lll}
\hline $\mathrm{T}\left[{ }^{\circ} \mathrm{C}\right]$ & $\mathrm{F}[\mathrm{N}]$ & $\mathrm{U}_{\mathrm{p}}[\mathrm{J}]$ \\
\hline 25 & 3096.17 & 30.31 \\
-55 & 2527.69 & 23.91 \\
70 & 2534.42 & 27.31
\end{tabular}

The higher rigidity is also denoted by the relatively higher slope of the first linear part of the load-displacement curves at a lower temperature (Fig.1). However, it is essential to note that in correspondence of maximum load, after the first 
Analysis of the extreme temperature's effect on the low-velocity impact behaviour and d...

excellent load drop (which denotes a significant breakage of the fibres) the load is maintained for a specific range of displacement, $\mathrm{d}$, at Ta and, even more so, at $\mathrm{T}=70^{\circ} \mathrm{C}$. The test temperature nearness justifies this behaviour to the PVC core's glass transition temperature $\left(\mathrm{Tg}=81^{\circ} \mathrm{C}\right)$. So, in the softening phase, the material is more willing to follow the movement of the penetrator, allowing a greater absorption of energy that sprays the highest recorded Up value. This does not happen at low temperatures where the global stiffening of the CFRP ply and the PVC core lead to a greater propensity to damage.

Figure 2 shows only the displacement force curves at $U=10 \mathrm{~J}$ for the three temperatures tested. As it is easy to observe, the resulting curves highlight the non-breaking of the sample. The area enclosed by the curves is representative of the energy absorbed by the material. This parameter is an index of the global damage obtained in the sandwich structure after impact: the greater the absorbed energy, Ua, the greater the damage. Damage can be evolved in an indentation, I, or in the form of delamination area,A. The first is the plastic deformation impress by the indenter on the impacted surface. The delamination area is representative of the fracture into layers because the adhesion between layers often fails to cause layers. From a first analysis of the curves, it is possible to observe that the absorbed energy increases as the temperature increases. This is a predictable result due to the epoxy being more ductile at higher temperatures, and more ductility leads to an increase in plastic deformation and viscous dissipation phenomena. Therefore at higher temperatures, there is less elasticity deformation energy that can be returned to the firing pin, resulting in greater absorbed energy values.

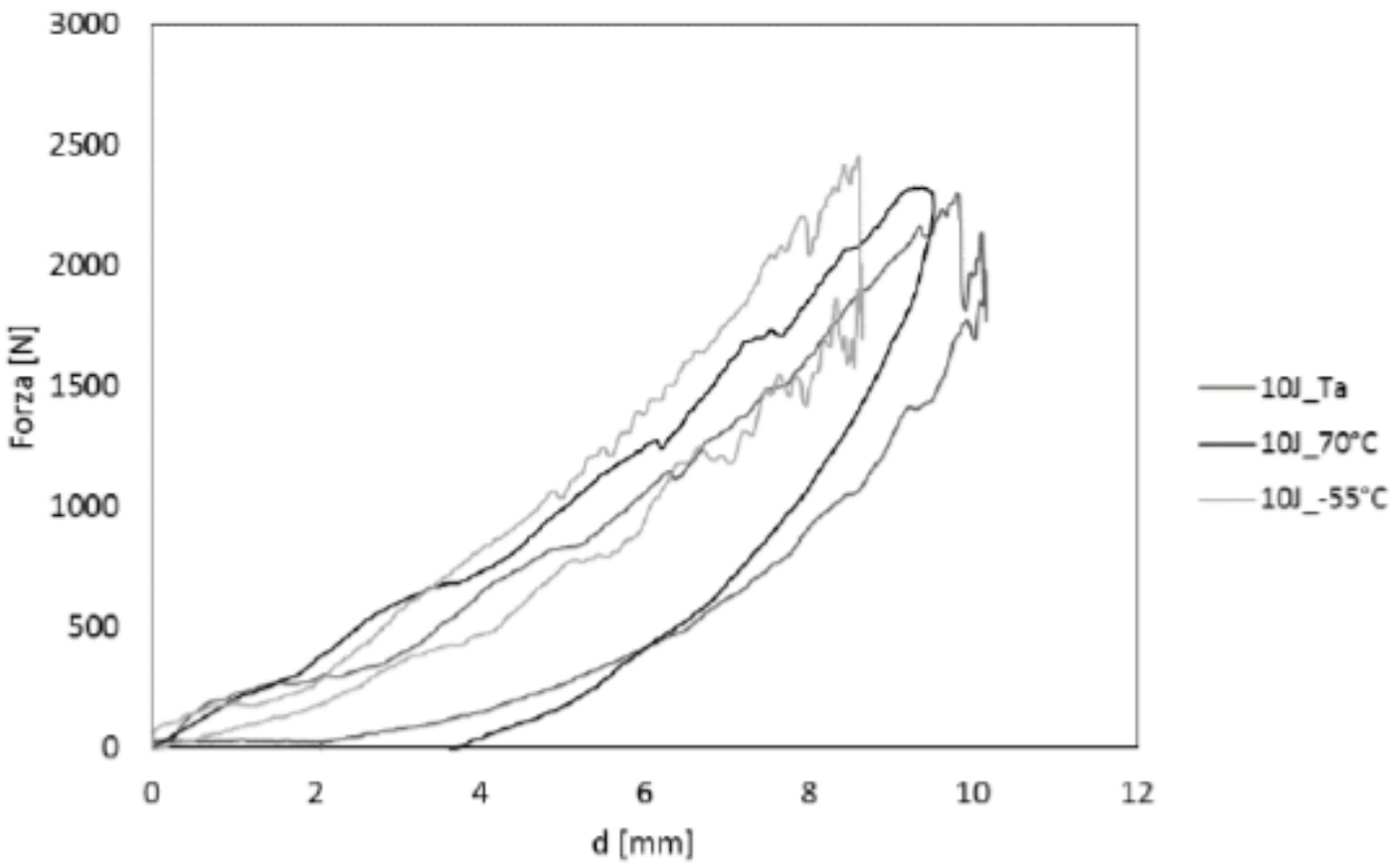

Fig. 2. Load-displacement curves at $\mathrm{U}=10 \mathrm{~J}$ for the three tested temperatures

From table 1, for a more detailed analysis, it is possible to evaluate the impact parameters obtained from the impact tests at $U=7.5 \mathrm{~J}, 10 \mathrm{~J}$ and 12J. From the parameters' values is clear that the influence of temperature on the impact behaviour of the laminates considered is evident. As the temperature increases, the material's energy absorption capacity, Ua, increases due to influence of core viscous flow, and this justifies the higher indentation depth, I, even if the maximum value of the impact force does not undergo significant variations with respect to the room temperature.

Table 1. Impact parameters and damage extension for all temperature and impact energy tested 


\begin{tabular}{lllllllllllll}
\hline & \multicolumn{3}{c}{$\mathrm{T}=70^{\circ} \mathrm{C}$} & \multicolumn{3}{c}{$\mathrm{T}=25^{\circ} \mathrm{C}$} & \multicolumn{4}{c}{$\mathrm{T}=55^{\circ} \mathrm{C}$} \\
\hline $\mathrm{U}[\mathrm{J}]$ & $\mathrm{F}_{\max }[\mathrm{N}]$ & $\mathrm{Ua}[\mathrm{J}]$ & $\mathrm{d}[\mathrm{mm}]$ & $\mathrm{I}[\mu \mathrm{m}]$ & $\mathrm{F}_{\max }[\mathrm{N}]$ & $\mathrm{Ua}[\mathrm{J}]$ & $\mathrm{d}[\mathrm{mm}]$ & $\mathrm{I}[\mu \mathrm{m}]$ & $\mathrm{F}_{\max }[\mathrm{N}]$ & $\mathrm{Ua}[\mathrm{J}]$ & $\mathrm{d}[\mathrm{mm}]$ & $\mathrm{I}[\mu \mathrm{m}]$ \\
\hline 7.5 & 1935.6 & 3.13 & 7.90 & 144.53 & 1905.20 & 2.04 & 8.66 & 84.83 & 2184.90 & 0.81 & 7.64 & 65.49 \\
\hline 10 & 2323.20 & 5.62 & 8.52 & 174.41 & 2297.09 & 4.96 & 10.15 & 106.26 & 2574.36 & 2.65 & 8.65 & 73.54 \\
\hline 12.5 & 2632.44 & 9.07 & 9.94 & 652.26 & 2521.40 & 7.94 & 10.32 & 301.51 & 2601.3 & 3.309 & 9.69 & 125.33 \\
\hline
\end{tabular}

On the other hand, as the temperature decreases, it is noted that the material stiffens and it has a reduced capacity to absorb the impact energy. The maximum force, Fmax, assumes higher values for the lower temperature values and a greater extent of the damage (figures 3 and 4). By observing figure 4 which correlates the indentation value, I, to the impact energies tested for the three temperatures, it is possible to easily verify that the plastic deformation increases as the energy and operating temperatures increase. In particular, the rise in indentation with increasing temperature is evident, the greater the impact of energy. At higher energies, the impact affects the external faces and the PVC core, which absorbs most of the energy. On the other hand, in figure 5 which correlates the delaminated area, A, identified by means of the $\mathrm{C}$-scan analysis with the impact energy, $\mathrm{U}$, it is noted that the damage increases as the temperature decreases, showing a smaller difference in correspondence of higher energy levels. In general, from the results, it seems that at low temperatures $\left(-55^{\circ} \mathrm{C}\right)$, the delaminated area is more evident and affects more the surface of the sampleOn the other hand, the impact is better absorbed at higher temperatures on the surface. Still, it seems to involve more large sub-surface layers resulting in the greater indentation.

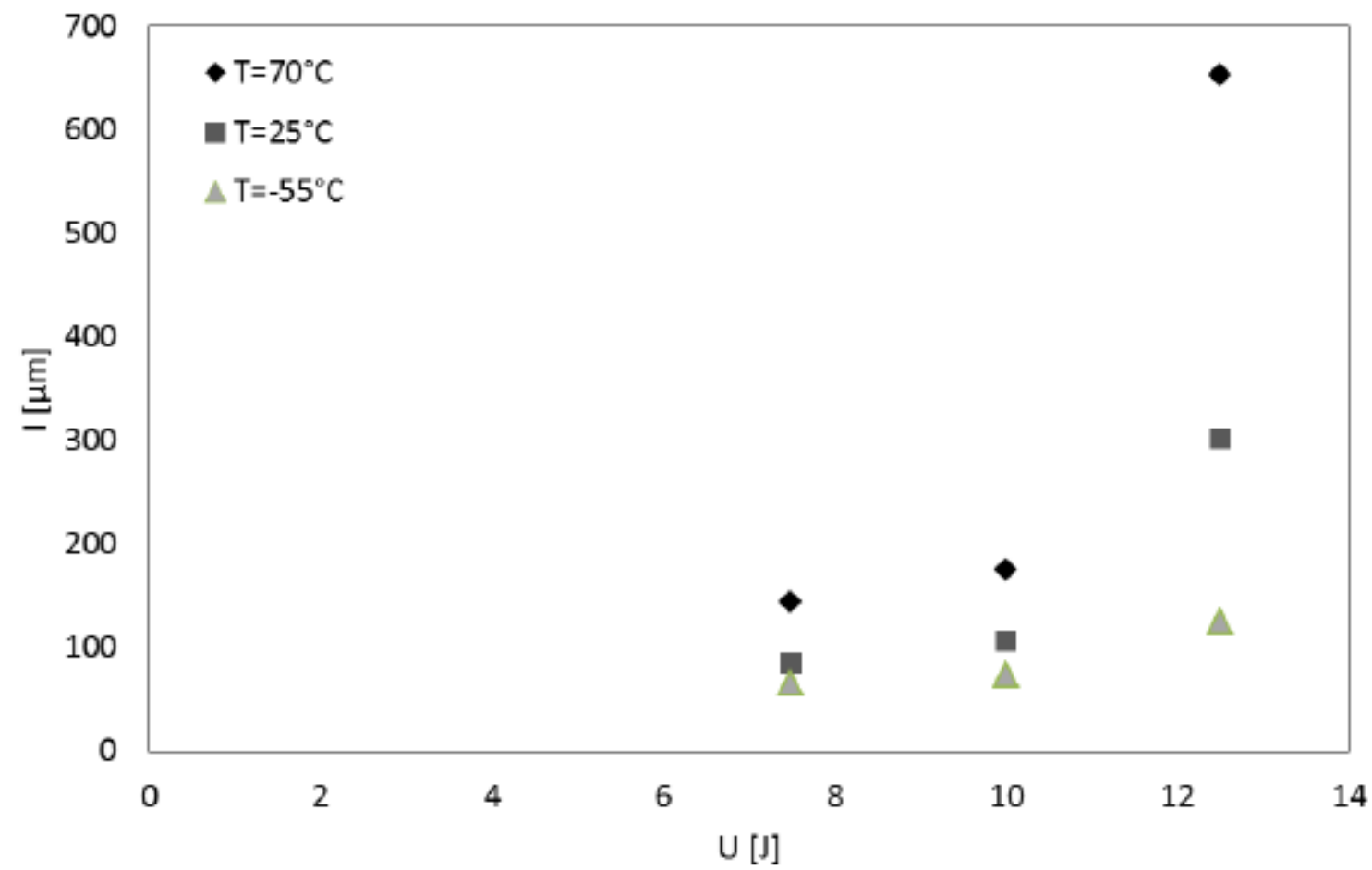

Fig. 4. Indentation depth, I, vs impact energy, $\mathrm{U}$ for all tested temperatures 


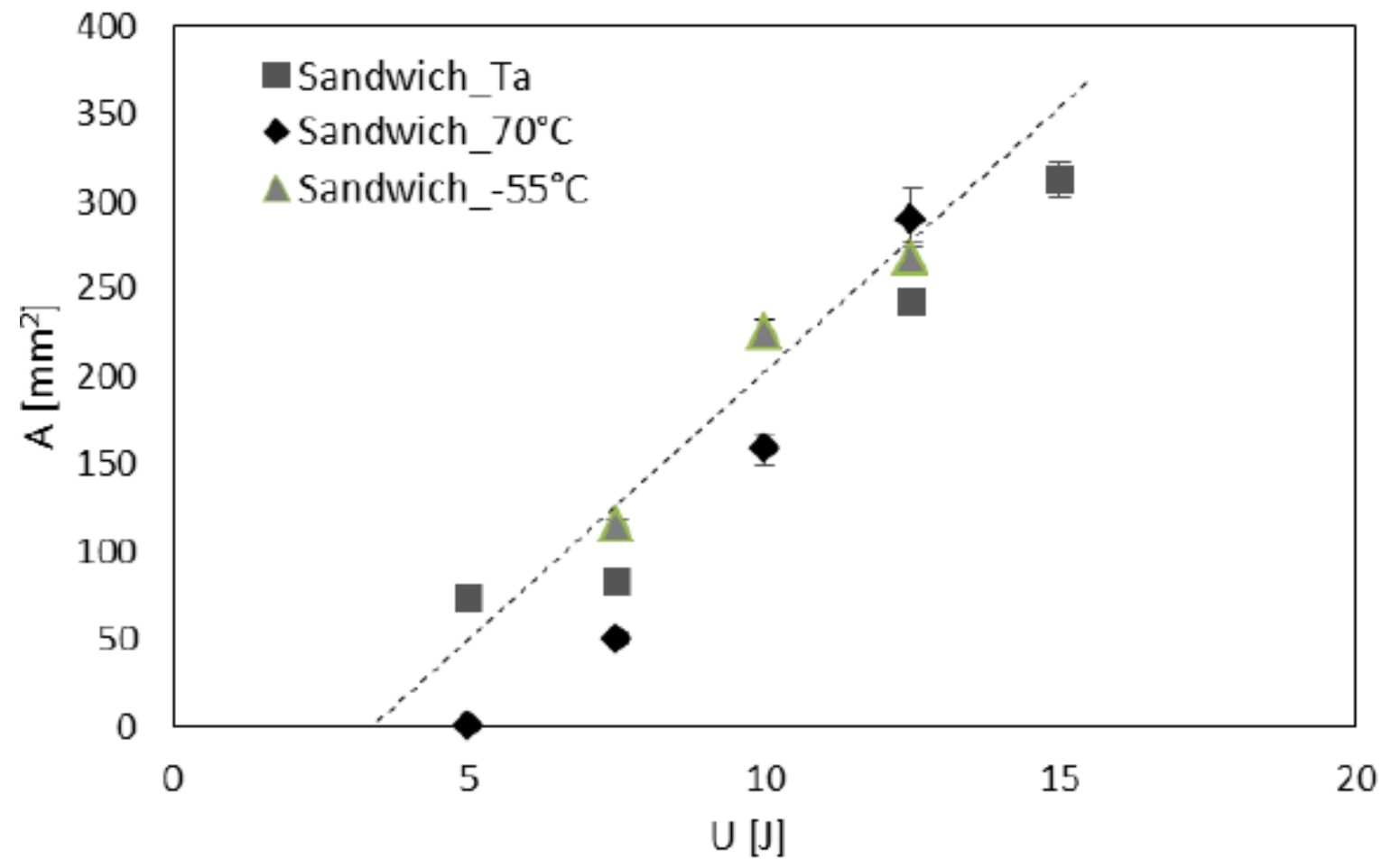

Fig. 5. Damage area, A, vs impact energy, U for all tested temperatures

The polymeric core's presence has an impact on energy absorption function, especially in high-temperature conditions where the plasticization of PVC results to improve it. At low temperatures, the core rigidly transmits the load to the lower face with its cracking.

The greater "risk" of low temperature impact is evidenced by the lower value assumed by the visible damage such as the indentation which represents footprint impress on the material by the impactor and the presence of greater extensive damage, A, within the laminate than the which ones detected at high temperatures. However, en exciting result can be observed from figure 5: all the data converge to a single linear master curve, independently of the temperature. The result is exciting since the useful and simple correlation between internal damage and impact energy, $\mathrm{U}$, suggests a useful way to know the internal damage, not visually inspectable as discussed and confirmed above, from a simple external measurement of the indentation I. So, it seems that for impact energy, U, less than 3.7J no delamination can be detected. Of course, several data needed to confirm this assertion.

\section{Conclusions}

In this paper, the effect of extreme temperature $\left(\mathrm{T}=-55^{\circ} \mathrm{C}, 70^{\circ} \mathrm{C}, 25^{\circ} \mathrm{C}\right)$ on sandwich composites' low velocity impact behaviour has been studied. The sandwiches structures made of CFRP face sheets and PVC core have been tested at three different energy levels ( $U=7.5 \mathrm{~J}, 10 \mathrm{~J}, 12.5 \mathrm{~J}$ ) for the three temperature analysed. After each impact test, the indentation measurement by confocal microscope and the delamination area by C-scan analysis have been evaluated to study the influence of temperature on the damage mode.

In general, better low-velocity impact performances are shown at room temperature denoting at a lower temperature $\left(\mathrm{T}=-55^{\circ} \mathrm{C}\right)$ a higher structure propensity to damage.

At indentation, in general, it seems that at low temperatures $\left(-55^{\circ} \mathrm{C}\right)$ the delaminated area is more evident and affects more the surface of the sample. On the other hand, while at higher temperatures the impact is better absorbed on the 
surface but seems to affect more large sub-surface layers resulting in greater indentation: the polymeric core's presence has an impact energy absorption function. The result is very interesting in terms of delamination since a useful and straightforward correlation between external damage and impact energy was found. This correlation suggests a useful way to know the internal damage, not visually inspectable, understanding the energy of the impact. It seems to exist a U value (3.7J) below the wich no delamination occurs. In the future, it would be necessary to have more and more data to assert the last consideration and predict the residual compression strength to assess how the different sandwiches tests temperatures can influence the mechanisms of failure.

\section{Acknowledgements}

The work was carried out within the TABASCO project - Campania DAC Aerospace Technological District funded by the Campania Region

\section{Bibliography}

[1] Lopresto, V. Leone, C. Caprino, G. De Iorio, I. Innovative sandwiches for civil applications. Procedia Eng. 2011;10:2058-67.

[2] Kwang, B.S. Jae, Y.L. Se, H,C, An experimental study of low-velocity impact responses of sandwich panels for Korean low floor bus. Composite Structures Volume 84, Issue 3, July 2008, Pages 228-240

[3] Reddy, T.Y., Wen, H.M., Reid, S.R. and Soden, P.D. (1998). Penetration and Perforation of Composite Sandwich Panels by Hemispherical and Conical Projectiles, Journal of Pressure Vessel Technology, 120: 186-194.

[4] Vaidya, U.K., Kamath, M.V., Hosur, M.V., Mahfuz, H. and Jeelani, S. (1999). Low- Velocity Impact Response of Cross-Ply Laminated Sandwich Composites with Hollow and Foam-Filled Z-Pin Reinforced Core, Journal of Composites Technology and Research, 21(2): 84-97.

[5] Yang, P. Shams, S. Slay, A. Brokate, B. Elhajjar, R. Evaluation of temperature effects on low velocity impact damage in composite sandwich panels with polymeric foam cores. Composite Structures 129 (2015) 213-223

[6] Erickson, M. D., Kallmeyer, A. R., \& Kellogg, K. G. (2005). Effect of Temperature on the Low-velocity Impact Behavior of Composite Sandwich Panels. Journal of Sandwich Structures \& Materials, 7(3), 245-264. doi:10.1177/ 1099636205048636

PDF automatically generated on 2021-05-22 03:43:04

Article url: https://popups.uliege.be/esaform21/index.php?id=2616

published by ULiège Library in Open Access under the terms and conditions of the CC-BY License (https://creativecommons.org/licenses/by/4.0) 